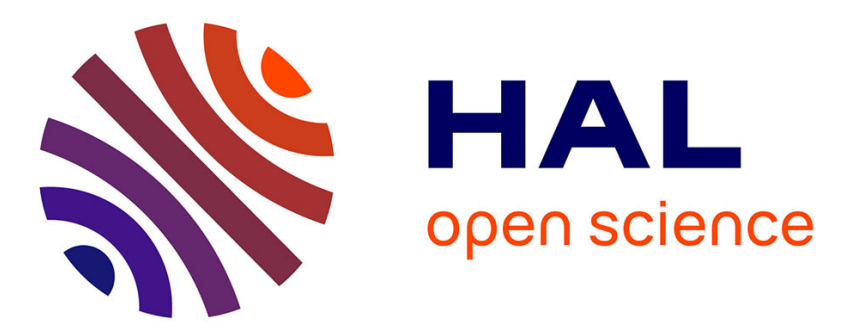

\title{
Accuracy of the tibiofemoral contact forces estimated by a subject-specific musculoskeletal model with fluoroscopy-based contact point trajectories
}

Raphaël Dumas, Florent Moissenet

\section{- To cite this version:}

Raphaël Dumas, Florent Moissenet. Accuracy of the tibiofemoral contact forces estimated by a subject-specific musculoskeletal model with fluoroscopy-based contact point trajectories. Journal of Biomechanics, 2020, 113, pp 110-117. 10.1016/j.jbiomech.2020.110117 . hal-03012368

\author{
HAL Id: hal-03012368 \\ https://hal.science/hal-03012368
}

Submitted on 18 Nov 2020

HAL is a multi-disciplinary open access archive for the deposit and dissemination of scientific research documents, whether they are published or not. The documents may come from teaching and research institutions in France or abroad, or from public or private research centers.
L'archive ouverte pluridisciplinaire $\mathbf{H A L}$, est destinée au dépôt et à la diffusion de documents scientifiques de niveau recherche, publiés ou non, émanant des établissements d'enseignement et de recherche français ou étrangers, des laboratoires publics ou privés. 


\section{Journal Pre-proofs}

Accuracy of the tibiofemoral contact forces estimated by a subject-specific musculoskeletal model with fluoroscopy-based contact point trajectories

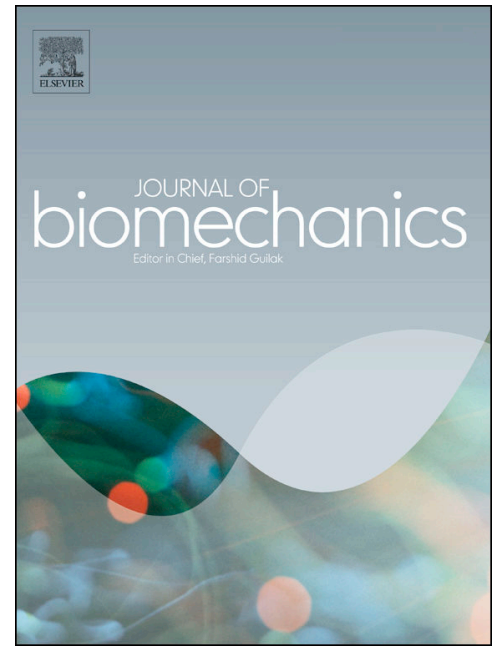

To appear in: $\quad$ Journal of Biomechanics

Received Date: $\quad 30$ January 2020

Revised Date: 22 October 2020

Accepted Date: $\quad 29$ October 2020

Please cite this article as: R. Dumas, F. Moissenet, Accuracy of the tibiofemoral contact forces estimated by a subject-specific musculoskeletal model with fluoroscopy-based contact point trajectories, Journal of Biomechanics (2020), doi: https://doi.org/10.1016/j.jbiomech.2020.110117

This is a PDF file of an article that has undergone enhancements after acceptance, such as the addition of a cover page and metadata, and formatting for readability, but it is not yet the definitive version of record. This version will undergo additional copyediting, typesetting and review before it is published in its final form, but we are providing this version to give early visibility of the article. Please note that, during the production process, errors may be discovered which could affect the content, and all legal disclaimers that apply to the journal pertain.

(C) 2020 Published by Elsevier Ltd. 


\title{
Accuracy of the tibiofemoral contact forces estimated
}

by a subject-specific musculoskeletal model

with fluoroscopy-based contact point trajectories

\author{
Raphael Dumas ${ }^{1}$, Florent Moissenet ${ }^{2}$ \\ ${ }^{1}$ Univ Lyon, Univ Gustave Eiffel, LBMC UMR_T 9406, F-69622, Lyon, France \\ ${ }^{2}$ Willy Taillard Laboratory of Kinesiology, University Geneva Hospitals and Geneva University, \\ Geneva, Switzerland
}

Word count: 2373 (introduction to discussion) 


\section{Abstract}

Accurate estimation of the tibiofemoral contact forces relies on exact kinematics and joint geometry. Subject-specific kinematic constraints representing contact point trajectories derived from fluoroscopic measurements during lunge are introduced in a musculoskeletal model of the lower limb and compared to generic kinematic constraints. The medial, lateral and total contact forces during gait and squat are validated using the data of four patients with an instrumented prosthesis. The accuracy of the estimated contact forces (both with subject-specific and generic kinematic constraints) remains close to the level reported in the literature. The mean root mean square errors range from 0.32 to 0.52 body weights for gait and from 0.27 to 0.72 body weights for squat. The impact of the subject-specific contact point trajectories is not found substantial or consistent between patients and tasks. Indeed, the kinematics of the total knee prostheses remains close to the kinematics of a hinge joint and the contact point locations remain generally centred at $20 \mathrm{~mm}$ from the tibia centreline (close to the constant value defined in the generic constraints). The contact point trajectories are also suspected to differ between tasks (lunge vs. gait and squat). While the contact point trajectories have been reported to be sensitive model parameters, no clear improvement of the contact force accuracy is demonstrated on patients with instrumented prosthesis. The introduction (as kinematic constraints) of fluoroscopy-based contact point trajectories may be considered in cases where these trajectories are significantly altered, as reported for osteoarthritis patients.

Keywords: tibiofemoral contact force, contact point, kinematics, personalisation, validation, instrumented prosthesis, gait, squat 


\section{Introduction}

Accurate estimation of the tibiofemoral contact forces relies on exact kinematics and joint geometry. Several subject-specific musculoskeletal models based on fluoroscopy data have been developed in the past. The measured tibiofemoral kinematics was directly applied to the model (Zheng et al., 2014) or translated into spline functions coupling the joint degrees of freedom (DoFs) (Gerus et al., 2013). To estimate the medial and lateral contact forces in addition to the total tibiofemoral contact forces, the contact point trajectories are also required and appear to be sensitive parameters (Lerner et al., 2015; Saliba et al., 2017; Zeighami et al., 2018). These contact point trajectories, translated into kinematic constraints, have been recently personalised using biplane radiography acquired during quasi-static squat movements (Zeighami et al., 2018), but not using fluoroscopy acquired during dynamic tasks. Furthermore, the accuracy of musculoskeletal models with subject-specific contact point trajectories has not been evaluated yet.

Such validation requires a comprehensive dataset (e.g. Grand Challenge Competition to Predict In Vivo Knee Loads (Fregly et al., 2012), CAMS-knee project (Taylor et al., 2017)) where fluoroscopy was performed during dynamics tasks on patients with an instrumented prosthesis. All CAMS-knee patients had fluoroscopy measurements (synchronised with marker motion and ground reaction measurements) during different dynamic tasks, while two Grand Challenge patients (JW and PS) had fluoroscopy measurements during treadmill gait. For all these measurements, fluoroscopy was mono-plane which makes the estimation of contact point trajectories difficult unless relying on an auxiliary computational contact model (Zhao et al., 2007). Though, biplane fluoroscopy measurements have been also reported during lunge and chair rising-sitting for three Grand Challenge patients (DM, SC, and PS) (Varadarajan et al., 2008). 
The objective of this study is therefore to validate the medial, lateral and total tibiofemoral

contact forces estimated during over-ground gait and squat by a musculoskeletal model with subject-specific contact point trajectories derived from the fluoroscopy measurements performed on the four Grand Challenge patients. It is hypothesised that the accuracy of the contact forces can be improved by these subject-specific contact point trajectories compared to generic ones.

\section{Material and Methods}

\section{Subject-specific contact point trajectories}

The characteristics of the four Grand Challenge patients (Fregly et al., 2012; Varadarajan et al., 2008; Zhao et al., 2007) including their age at the time of fluoroscopy measurements and motion capture (marker motion, ground reaction and electromyography measurements) are displayed in Table 1 . The implants were made of a posterior cruciate-retaining femoral component and a standard congruent fixed-bearing tibial insert. For one patient (JW), the prosthesis kinematics during treadmill gait and the contact point trajectories resulting from a computational contact model are taken from Zhao et al. (2007). The prosthesis kinematics was measured using monoplane fluoroscopy. An accuracy of $1.1 \mathrm{deg}$ and of $0.5 \mathrm{~mm}$ (in plane) to $6.6 \mathrm{~mm}$ (out of plane) was reported by the authors (Banks and Hodge, 1996). The contact points correspond to the centres of pressure of an elastic foundation model driven by the fluoroscopy-based kinematics (flexion-extension, internal-external rotation, and anteriorposterior displacement only), with a maximal knee flexion reaching $65 \mathrm{deg}$. For the three other patients (DM, SC, and PS), the prosthesis kinematics and the contact point trajectories are digitised from curves displaying one cycle of knee lunge (Varadarajan et al., 2008), with a maximal knee flexion reaching 85 to $95 \mathrm{deg}$. The prosthesis kinematics was measured using 
bi-plane fluoroscopy. An accuracy of $0.05 \mathrm{deg}$ and $0.38 \mathrm{~mm}$ was reported by the authors. The contact points correspond directly to the centroids of the intersection area between each femoral condyle and polyethylene articular surfaces (tibial inserts). The contact point trajectories on the tibial inserts are illustrated in Figure 1.

\section{Musculoskeletal model}

A 3D lower limb musculoskeletal model made of five segments (pelvis, thigh, patella, shank and foot), five joint DoFs, and forty-three muscle lines of action (Moissenet et al., 2014; Zeighami et al., 2018) is used in this study. For the tibiofemoral joint, subject-specific kinematic constraints are introduced in the musculoskeletal model as the position of the contact points on the medial and lateral condyles and plateaus, expressed in the thigh and shank segment coordinate systems as a function of the tibiofemoral flexion angle (Zeighami et al., 2018). For that, the positions of the femoral component and tibial insert with respect to the corresponding bones are obtained from the four patient CT-scans (Fregly et al., 2012). Subject-specific patellofemoral kinematic constraints (hinge joint and isometric patellar tendon) are also derived from the CT-scans. For one of the patients (PS), this modelling approach showed errors of $13 \mathrm{deg}$ in flexion, 11 and $5 \mathrm{~mm}$ in anterior and superior displacements during unloaded leg-swing (Marra et al., 2015).

For comparison, generic kinematic constraints are built to represent generic couplings between joint DoFs and two contacts at $20 \mathrm{~mm}$ from the tibia centreline (Gerus et al., 2013; Zeighami et al., 2018). These generic couplings are based on an ellipse moving on a line with specified contact points (Delp et al., 1990). The polynomials defining the subject-specific and generic kinematic constraints are provided in Supplementary Table S1. 
The muscular geometry was scaled based on the average of the thigh and shank lengths. After inverse kinematics with either the subject-specific or the generic kinematic constraints, the musculo-tendon forces and contact forces are computed by minimising simultaneously the sum of the squared activations and the sum of the squared hip, knee and ankle contact forces.

On the same patients, with generic kinematic constraints, a similar simultaneous minimisation has been reported to provide accurate muscle excitations and total tibiofemoral contact forces (Zargham et al., 2019). Minimisation of the contact forces was also introduced in the model calibration to obtain accurate tibiofemoral contact force predictions for patient JW (Gerus et al., 2013) and patient DM (Lerner et al., 2015). In the present study, hip, patellofemoral, medial and lateral tibiofemoral and ankle contact forces are normalised by 3, $1,1.5,1.5$, and 3 body weights (BW) respectively to be aggregated with the musculo-tendon activations. Thanks to the formulation of the kinematic constraints, the medial and lateral tibiofemoral contact forces (corresponding to Lagrange multipliers (Zeighami et al., 2018)) are directly computed along the contact point trajectories while considering a 3D joint equilibrium. Hip, patellofemoral, and ankle contact forces are also computed through Lagrange multipliers (Moissenet et al., 2014) but are not specifically investigated in the present validation study.

\section{Statistics}

Based on five gait cycles and four squat cycles for each patient, mean and standard deviation of errors on peak forces, root mean square errors (RMSE) and coefficients of determination $\left(R^{2}\right)$ are computed between the estimated and the measured contact forces. In addition, concordance coefficients ( $C$, percentage of phases with matched active/inactive states) are computed between the estimated musculo-tendon forces and the measured 
maximus, gluteus medius, adductor magnus, vastus medialis, vastus lateralis, rectus femoris, semimembranosus, biceps femoris long head, tensor fascia latae, gastrocnemius medialis, gastrocnemius lateralis, soleus, tibialis anterior, and peroneus longus. To do so, the gait cycle is divided into seven phases (loading response to terminal swing) and the squat cycle is divided into three phases (descent, maximum flexion, and ascent). Inverse kinematics marker residuals are also assessed.

\section{Results}

With the fluoroscopy-based contact point trajectories, the mean RMSEs of the total contact force across gait cycles and patients range from 0.32 to $0.52 \mathrm{BW}$ and the mean coefficients of determination range from 0.71 to 0.92 . For squat, mean RMSEs and $\mathrm{R}^{2}$ range between 0.27 and $0.72 \mathrm{BW}$ and between 0.53 and 0.95 . This personalisation did not systematically improves the accuracy of contact forces (Figures $2 \& 3$, Tables $2 \& 3$ ) and the mean differences between subject-specific and generic kinematic constraints on the peaks is $0.14 \mathrm{BW}$ for gait and 0.50 BW for squat. Inverse kinematics marker residuals remains between 10 and $24 \mathrm{~mm}$ for gait and between and 14 and $37 \mathrm{~mm}$ for squat. The estimation of musculo-tendon forces remains mostly with the same level of concordance (C from $58.7 \%$ to $73.2 \%$ ).

\section{Discussion}

The objective of this study is to validate the tibiofemoral contact forces obtained from a musculoskeletal model with subject-specific contact point trajectories. The kinematic constraints derived from fluoroscopic measurements are introduced in the model at both inverse kinematics and inverse dynamics steps and, therefore, impact the computation in 
several ways. First, the joint kinematics is modified (Supplementary Figures S1 \& S2). This implies a slightly altered knee joint position with respect to the force plates and thus altered external flexion-extension and adduction-abduction moments. Second, the muscle lever arms are directly changed (Gerus et al., 2013; Koo and Koo, 2019) as well as musculo-tendon lengths and velocities. Third, the contact points where the contact forces are computed are also directly changed (Dumas et al., 2020b; Zeighami et al., 2018). As consequence, musculotendon forces, total tibiofemoral contact force and its distribution between the medial and lateral compartments are potentially all impacted. The hypothesis that the accuracy of the contact forces is improved by subject-specific contact point trajectories cannot be confirmed. Indeed, the impact of the subject-specific contact point trajectories is not found substantial or consistent between patients and tasks.

It can be understood that the generic kinematic constraints generally provide a fair representation of the studied prosthesis kinematics and contact point trajectories and, therefore, only minor improvements could be expected. Indeed, it was already shown for patient PS that accurate contact forces can be predicted during gait with a simple hinge joint model at the knee (Marra et al., 2015). Additionally, it was shown for patient DM that the sensitivity of the contact force to contact location is limited to $0.06 \mathrm{BW} / \mathrm{mm}$ (Lerner et al., 2015). In the present study, the contact point locations, except for the lateral compartment of patient SC, remain centred around $20 \mathrm{~mm}$ from the tibia centreline (Figure 1), i.e. close to the constant value defined in the generic kinematic constraints. The polynomial constants for the position of the contact point about the Z-axis (Supplementary Table S1) range between 18 and $22 \mathrm{~mm}$ in absolute values (16 and $30 \mathrm{~mm}$ for patient $\mathrm{SC}$ ). The impact of the kinematic constraints on the marker residuals is also marginal. 
Moreover, it is possible that the contact point trajectories observed during lunge are not replicated during gait and squat (as well as between treadmill and over-ground gait for patient JW). Yet, the contact point trajectories were not really different between the lunge and chair rising-sitting movements (Varadarajan et al., 2008). The lunge movement has been preferred to the chair rising-sitting movement because it demonstrated, on patient DM, PS, and SC, higher and better balanced contact forces between medial and lateral compartments, more similar to the ones observed during treadmill gait for patient (JW) (Zhao et al., 2007). It is important to note that the two sessions (lunge vs. gait and squat) were separated of 2 to 4 years in time (Table 1). Still, using inverse kinematics, the kinematics derived from the subjectspecific contact point trajectories (trajectories obtained during lunge movement two years before) and the kinematics measured during treadmill gait for patient PS compare favorably (Supplementary Figure S1).

The accuracy of the estimated total contact force (both with subject-specific and generic kinematic constraints) during gait remains close to the level reported by Zargham et al. (2019) and does not reach the level of more advanced modelling (Chen et al., 2016; Gerus et al., 2013; Marra et al., 2015; Viceconti et al., 2019). On the same patients, a comparable level of concordance between estimated musculo-tendon forces and EMG signals (about 60\%) has been previously reported with other generic kinematic constraints (Moissenet et al., 2014). Using the CAMS-knee dataset, the total contact force accuracy of a generic musculoskeletal model was reported at $\mathbf{4 7 . 5 \%}$ for gait and $\mathbf{1 0 5 . 7 \%}$ for squat (Imani Nejad et al., 2020) while it ranges between $18.3 \%$ and $50.5 \%$ for gait and between $28.6 \%$ and $61.4 \%$ for squat in the present study.

The present study presents some limitations. Few model parameters out of many (and typically the muscle lines of action and muscle strength) have been personalised. The 
patellofemoral joint has been personalised based on CT-scans but with a simplified hinge model. Modelling of the patellofemoral joint as a hinge (generic or subject-specific) is common in musculoskeletal modelling (Barzan et al., 2019; Modenese et al., 2011; Xu et al., 2016) and was already applied on the Grand Challenge dataset (Marra et al., 2015; Moissenet et al., 2014; Viceconti et al., 2019). The present study exploits the only materials available to validate a musculoskeletal models with subject-specific contact point trajectories and is limited to four elderly patients. The contact point trajectories can differ between lunge, (treadmill and overground) gait and squat, and can vary from time to time. For patient JW, the contact point trajectories are defined during treadmill gait and are extrapolated for knee flexion exceeding 65 deg, which may provide unrealistic kinematics during squat (Supplementary Figure S2). Mobile fluoroscopy systems exist nowadays (Gray et al., 2019; Postolka et al., 2020) that can provide, simultaneous to motion capture, more representative contact point trajectories during over-ground gait or other tasks. Due to the study limitations, it is currently difficult to definitively conclude on the usefulness of the subject-specific kinematic constraints based on fluoroscopy-based contact point trajectories. Model personalisation increases complexity, processing time and costs and should only target sensitive model parameters. The contact forces are certainly sensitive to the contact point trajectories (Dumas et al., 2020b; Lerner et al., 2015; Nissan, 1980; Saliba et al., 2017; Zeighami et al., 2018) but no clear improvement of the accuracy could have been currently demonstrated. The CAMS-Knee dataset (with mobile mono-plane fluoroscopic measurements and simultaneous motion capture (Taylor et al., 2017) may help in demonstrating this improvement if an auxiliary computational contact model driven by the fluoroscopy-based kinematics (Zhao et al., 2007) is used to compute the contact point trajectories. This could be considered in a future study, also investigating 
different tasks (downhill walking, stair descent, sitting and rising from a chair) and another prosthesis design.

For validation purpose, the present study and the studies mentioned in perspective are performed on patients with total knee prostheses, but subject-specific contact point trajectories can be obtained the same way on patients or subjects without an implant (Gray et al., 2019; Koo and Koo, 2019). Typically, in the case of knee osteoarthritis, the contact point trajectories, among other joint and muscle factors, are modified by the pathology (Scarvell et al., 2018) and restored by the arthroplasty (Li et al., 2015). The alteration of the contact points appears to be a clinically relevant parameter to assess joint pathology (Farrokhi et al., 2014; Zeighami et al., 2017) as well as prosthetic performance (Gray et al., 2020; Nicolet-Petersen et al., 2020). For instance, the contact point trajectories can help estimating the in vivo slip velocity (Andriacchi et al., 2003; Dumas et al., 2020a; Hamilton et al., 2005) which is an important parameter, together with the estimated in vivo contact force, for characterising wear in the prosthetic components. These clinically relevant parameters could be profitably taken into account in the musculoskeletal model.

\section{Acknowledgements}

The authors would like to thank Benjamin J. Fregly from Rice University for providing detailed fluoroscopic and computational model results from Zhao et al. (2007) and Clément Marais from for Université de Lyon for his help in processing the kinematics and geometrical data. This work was partially founded by the LABEX PRIMES (ANR-11-LABX-0063) of Université de Lyon.

\section{Conflict of interest}


The authors do not have any financial or personal relationships with other people or

organisations that would have inappropriately influenced this study.

\section{References}

Andriacchi, T.P., Dyrby, C.O., Johnson, T.S., 2003. The Use of Functional Analysis in Evaluating Knee Kinematics. Clinical Orthopaedics and Related Research 410.

Banks, S.A., Hodge, W.A., 1996. Accurate measurement of three-dimensional knee replacement kinematics using single-plane fluoroscopy. IEEE Transactions on Biomedical Engineering 43, 638-649.

Barzan, M., Modenese, L., Carty, C.P., Maine, S., Stockton, C.A., Sancisi, N., Lewis, A., Grant, J., Lloyd, D.G., Brito da Luz, S., 2019. Development and validation of subject-specific pediatric multibody knee kinematic models with ligamentous constraints. Journal of Biomechanics 93, 194-203.

Chen, Z., Zhang, Z., Wang, L., Li, D., Zhang, Y., Jin, Z., 2016. Evaluation of a subject-specific musculoskeletal modelling framework for load prediction in total knee arthroplasty. Medical Engineering \& Physics 38, 708-716.

Delp, S.L., Loan, J.P., Hoy, M.G., Zajac, F.E., Topp, E.L., Rosen, J.M., 1990. An interactive graphics-based model of the lower extremity to study orthopaedic surgical procedures. IEEE Transactions on Biomedical Engineering 37, 757-767.

Dumas, R., Moissenet, F., Cheze, L., 2020a. Fluoroscopy-based subject-specific knee joint constraints for the estimation of prosthesis kinematics and contact velocities during gait. Computer Methods in Biomechanics and Biomedical Engineering, Accepted.

260 Dumas, R., Zeighami, A., Aissaoui, R., Year Knee Medial and Lateral Contact Forces Computed Along Subject-Specific Contact Point Trajectories of Healthy Volunteers and Osteoarthritic Patients. In Computer Methods, Imaging and Visualization in Biomechanics and Biomedical Engineering. Cham.

Farrokhi, S., Voycheck, C.A., Klatt, B.A., Gustafson, J.A., Tashman, S., Fitzgerald, G.K., 2014. Altered tibiofemoral joint contact mechanics and kinematics in patients with knee osteoarthritis and episodic complaints of joint instability. Clinical Biomechanics 29, 629-635. Fregly, B.J., Besier, T.F., Lloyd, D.G., Delp, S.L., Banks, S.A., Pandy, M.G., D'Lima, D.D., 2012. Grand challenge competition to predict in vivo knee loads. Journal of Orthopaedic Research 30, 503-513.

270 Gerus, P., Sartori, M., Besier, T.F., Fregly, B.J., Delp, S.L., Banks, S.A., Pandy, M.G., D'Lima, D.D., Lloyd, D.G., 2013. Subject-specific knee joint geometry improves predictions of medial tibiofemoral contact forces. Journal of Biomechanics 46, 2778-2786.

Gray, H.A., Guan, S., Thomeer, L.T., Schache, A.G., de Steiger, R., Pandy, M.G., 2019. Threedimensional motion of the knee-joint complex during normal walking revealed by mobile biplane x-ray imaging. Journal of Orthopaedic Research 37, 615-630.

Gray, H.A., Guan, S., Young, T.J., Dowsey, M.M., Choong, P.F., Pandy, M.G., 2020. Comparison of posterior-stabilized, cruciate-retaining, and medial-stabilized knee implant motion during gait. Journal of Orthopaedic Research 38, 1753-1768. 
Hamilton, M.A., Sucec, M.C., Fregly, B.J., Banks, S.A., Sawyer, W.G., 2005. Quantifying Multidirectional Sliding Motions in Total Knee Replacements. Journal of Tribology 127, 280286.

Imani Nejad, Z., Khalili, K., Hosseini Nasab, S.H., Schütz, P., Damm, P., Trepczynski, A., Taylor, W.R., Smith, C.R., 2020. The Capacity of Generic Musculoskeletal Simulations to Predict Knee Joint Loading Using the CAMS-Knee Datasets. Annals of Biomedical Engineering 48, 14301440.

Koo, Y.-J., Koo, S., 2019. Three-Dimensional Kinematic Coupling of the Healthy Knee During Treadmill Walking. Journal of Biomechanical Engineering 141.

Lerner, Z.F., DeMers, M.S., Delp, S.L., Browning, R.C., 2015. How tibiofemoral alignment and contact locations affect predictions of medial and lateral tibiofemoral contact forces. Journal of Biomechanics 48, 644-650.

Li, C., Hosseini, A., Tsai, T.-Y., Kwon, Y.-M., Li, G., 2015. Articular contact kinematics of the knee before and after a cruciate retaining total knee arthroplasty. Journal of Orthopaedic Research 33, 349-358.

Marra, M.A., Vanheule, V., Fluit, R., Koopman, B.H.F.J.M., Rasmussen, J., Verdonschot, N., Andersen, M.S., 2015. A Subject-Specific Musculoskeletal Modeling Framework to Predict In Vivo Mechanics of Total Knee Arthroplasty. Journal of Biomechanical Engineering 137.

Modenese, L., Phillips, A.T.M., Bull, A.M.J., 2011. An open source lower limb model: Hip joint validation. Journal of Biomechanics 44, 2185-2193.

Moissenet, F., Chèze, L., Dumas, R., 2014. A 3D lower limb musculoskeletal model for simultaneous estimation of musculo-tendon, joint contact, ligament and bone forces during gait. Journal of Biomechanics 47, 50-58.

Nicolet-Petersen, S., Saiz, A., Shelton, T., Howell, S.M., Hull, M.L., 2020. Small differences in tibial contact locations following kinematically aligned TKA from the native contralateral knee. Knee Surgery, Sports Traumatology, Arthroscopy 28, 2893-2904.

305 Nissan, M., 1980. Review of some basic assumptions in knee biomechanics. Journal of Biomechanics 13, 375-381.

Postolka, B., Schütz, P., Fucentese, S.F., Freeman, M.A.R., Pinskerova, V., List, R., Taylor, W.R., 2020. Tibio-femoral kinematics of the healthy knee joint throughout complete cycles of gait activities. Journal of Biomechanics 110, 109915.

310 Saliba, C.M., Brandon, S.C.E., Deluzio, K.J., 2017. Sensitivity of medial and lateral knee contact force predictions to frontal plane alignment and contact locations. Journal of Biomechanics 57, 125-130.

Scarvell, J.M., Galvin, C.R., Perriman, D.M., Lynch, J.T., van Deursen, R.W.M., 2018. Kinematics of knees with osteoarthritis show reduced lateral femoral roll-back and maintain an adducted position. A systematic review of research using medical imaging. Journal of Biomechanics 75, 108-122.

Taylor, W.R., Schütz, P., Bergmann, G., List, R., Postolka, B., Hitz, M., Dymke, J., Damm, P., Duda, G., Gerber, H., Schwachmeyer, V., Hosseini Nasab, S.H., Trepczynski, A., Kutzner, I., 2017. A comprehensive assessment of the musculoskeletal system: The CAMS-Knee data set. Journal of Biomechanics 65, 32-39.

Varadarajan, K.M., Moynihan, A.L., D'Lima, D., Colwell, C.W., Li, G., 2008. In vivo contact kinematics and contact forces of the knee after total knee arthroplasty during dynamic weightbearing activities. Journal of Biomechanics 41, 2159-2168. 
Viceconti, M., Ascani, D., Mazzà, C., 2019. Pre-operative prediction of soft tissue balancing in knee arthoplasty part 1: Effect of surgical parameters during level walking. Journal of Orthopaedic Research 37, 1537-1545.

Xu, C., Silder, A., Zhang, J., Hughes, J., Unnikrishnan, G., Reifman, J., Rakesh, V., 2016. An Integrated Musculoskeletal-Finite-Element Model to Evaluate Effects of Load Carriage on the Tibia During Walking. Journal of Biomechanical Engineering 138.

Zargham, A., Afschrift, M., De Schutter, J., Jonkers, I., De Groote, F., 2019. Inverse dynamic estimates of muscle recruitment and joint contact forces are more realistic when minimizing muscle activity rather than metabolic energy or contact forces. Gait \& Posture 74, 223-230. Zeighami, A., Aissaoui, R., Dumas, R., 2018. Knee medial and lateral contact forces in a musculoskeletal model with subject-specific contact point trajectories. Journal of Biomechanics 69, 138-145.

Zeighami, A., Dumas, R., Kanhonou, M., Hagemeister, N., Lavoie, F., de Guise, J.A., Aissaoui, R., 2017. Tibio-femoral joint contact in healthy and osteoarthritic knees during quasi-static squat: A bi-planar X-ray analysis. Journal of Biomechanics 53, 178-184.

Zhao, D., Banks, S.A., D'Lima, D.D., Colwell Jr, C.W., Fregly, B.J., 2007. In vivo medial and lateral tibial loads during dynamic and high flexion activities. Journal of Orthopaedic Research 25, 593-602.

Zheng, L., Li, K., Shetye, S., Zhang, X., 2014. Integrating dynamic stereo-radiography and surface-based motion data for subject-specific musculoskeletal dynamic modeling. Journal of Biomechanics 47, 3217-3221. 


\section{Figures and tables captions}

\section{Figure 1:}

Bottom views of the femoral components ( $45^{\circ}$ flexed) and top view of the tibial inserts with the contact point trajectories obtained during treadmill gait (mono-plane fluoroscopy and computational contact model (Zhao et al., 2007)) for patient JW and during lunge (biplane fluoroscopy (Varadarajan et al., 2008)) for patients DM, SC, and PS. X-axis denotes Anterior(+) / Posterior(-) direction and Z-axis denotes Lateral(+) / Medial(-) direction. Figures for patients SC and PS (with a left prosthesis) have been symmetrised.

Figure 2:

Mean and standard deviation of the medial, lateral and total contact forces in body weight (BW) measured by the instrumented prosthesis (red lines and areas (Fregly et al., 2012)), estimated with generic kinematic constraints (orange lines and areas), and estimated with subject-specific kinematic constraints (blue lines and areas) during gait. For interpretation of the references to colour in this figure legend, the reader is referred to the web version of this article.

Figure 3:

Mean and standard deviation of the medial, lateral and total contact forces in body weight (BW) measured by the instrumented prosthesis (red lines and areas (Fregly et al., 2012)), estimated with generic kinematic constraints (orange lines and areas), and estimated with subject-specific kinematic constraints (blue lines and areas) during squat. For interpretation 
of the references to colour in this figure legend, the reader is referred to the web version of this article.

Table 1:

Anthropometrics of the Grand Challenge patients.

Table 2:

Mean (and standard deviation) of the error on peak forces, root mean square errors (RMSE) and coefficients of determination $\left(R^{2}\right)$ between the estimated (with generic and subjectspecific kinematic constraints) and the measured medial, lateral, and total contact forces during gait. Mean (and standard deviation) of the concordance coefficients (C) between the estimated musculo-tendon forces and the measured electromyographic (EMG) signals. Mean (and standard deviation) of the markers residuals. Improvements are highlighted in bold.

Table 3:

Mean (and standard deviation) of the error on peak forces, root mean square errors (RMSE) and coefficients of determination $\left(R^{2}\right)$ between the estimated (with generic and subjectspecific kinematic constraints) and the measured medial, lateral, and total contact forces during squat. Mean (and standard deviation) of the concordance coefficients (C) between the estimated musculo-tendon forces and the measured electromyographic (EMG) signals. Mean (and standard deviation) of the marker residuals. Improvements are highlighted in bold. 


\section{Supplementary figure and table caption}

Polynomials (function of the tibiofemoral flexion) defining the generic and subject-specific kinematic constraints: position of the contact points on the medial and lateral condyles and plateaus, expressed in the femoral components and tibial inserts coordinate systems. The transformations from the femur (thigh segment) and tibia (shank segment) coordinate systems to the femoral components and tibial inserts coordinate systems are also provided.

\section{Supplementary Figure S1:}

Mean (and standard deviation) of the tibiofemoral kinematics obtained by inverse kinematics with the generic (orange lines and areas) and the subject-specific kinematic constraints (blue lines and areas) during gait.

For patient PS, the kinematics measured by mono-plane fluoroscopy during treadmill gait .(red circles of 143 frames (Fregly et al., 2012)) is also displayed. The correspondence between the percentages of the over-ground gait cycle and the treadmill gait frames is based on the mean flexion value.

Supplementary Figure S2:

Mean (and standard deviation) of the tibiofemoral kinematics obtained by inverse kinematics with the generic (orange lines and areas) and the subject-specific kinematic constraints (blue lines and areas) during squat. 


\section{Figure 1}
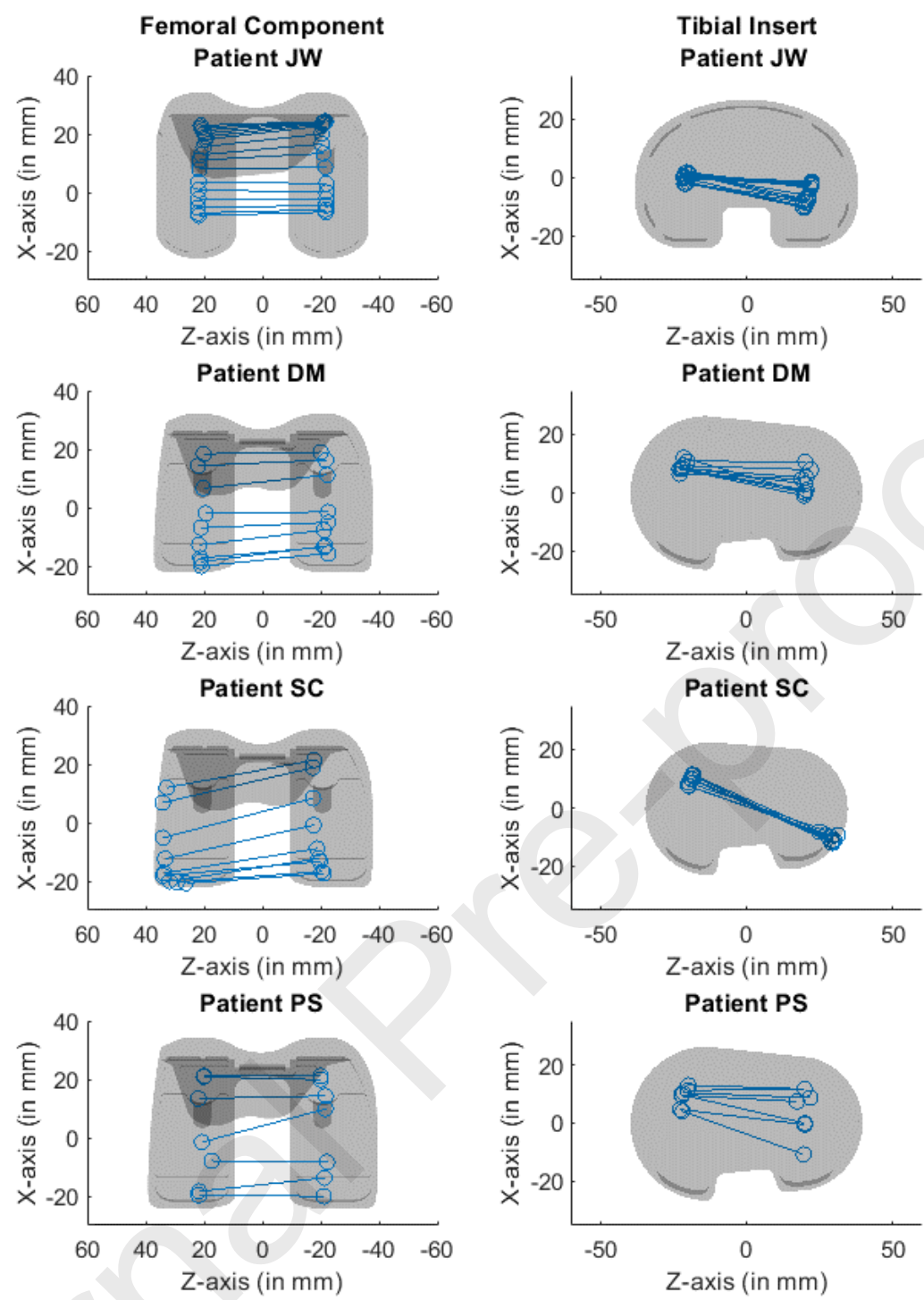
Figure 2
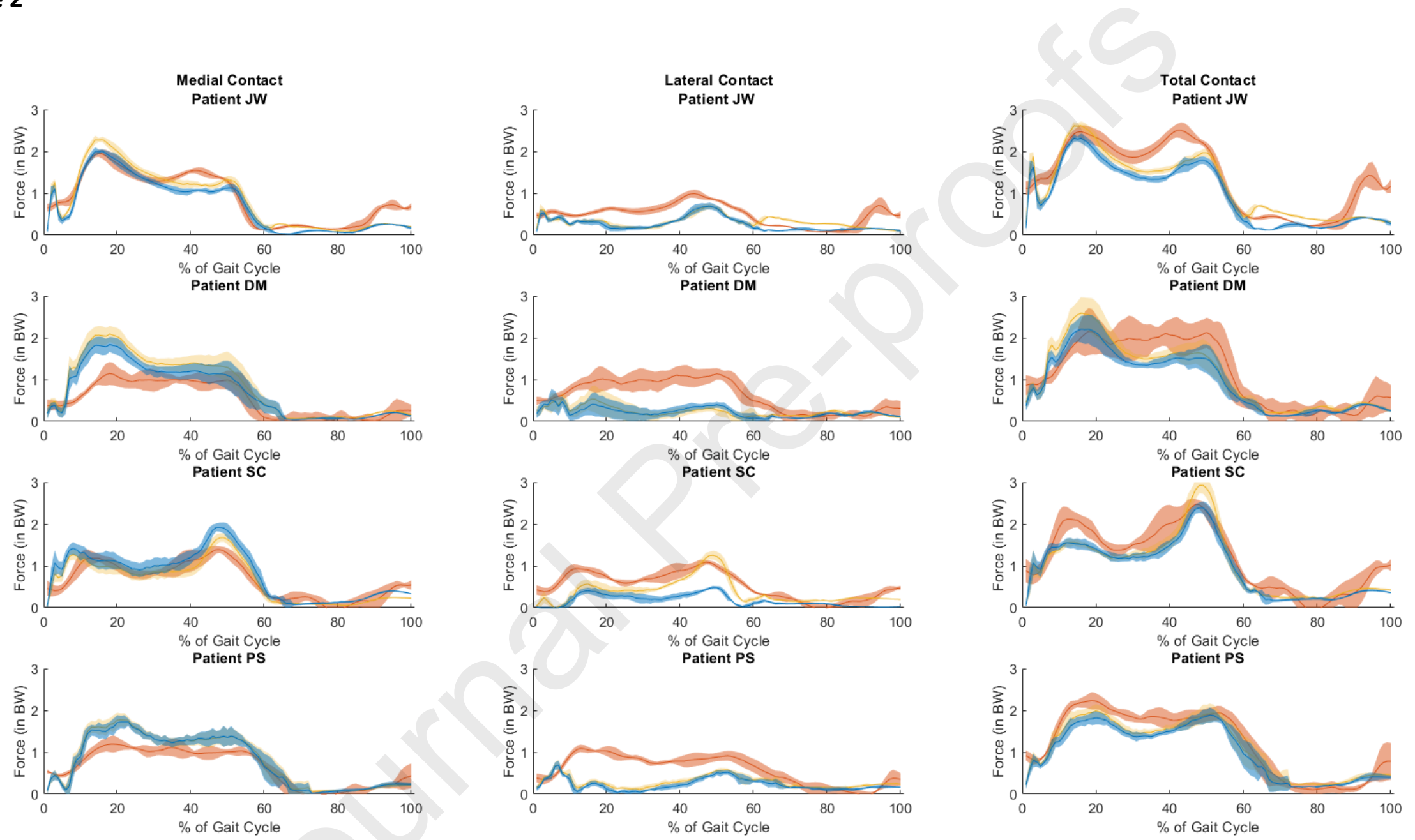
Figure 3
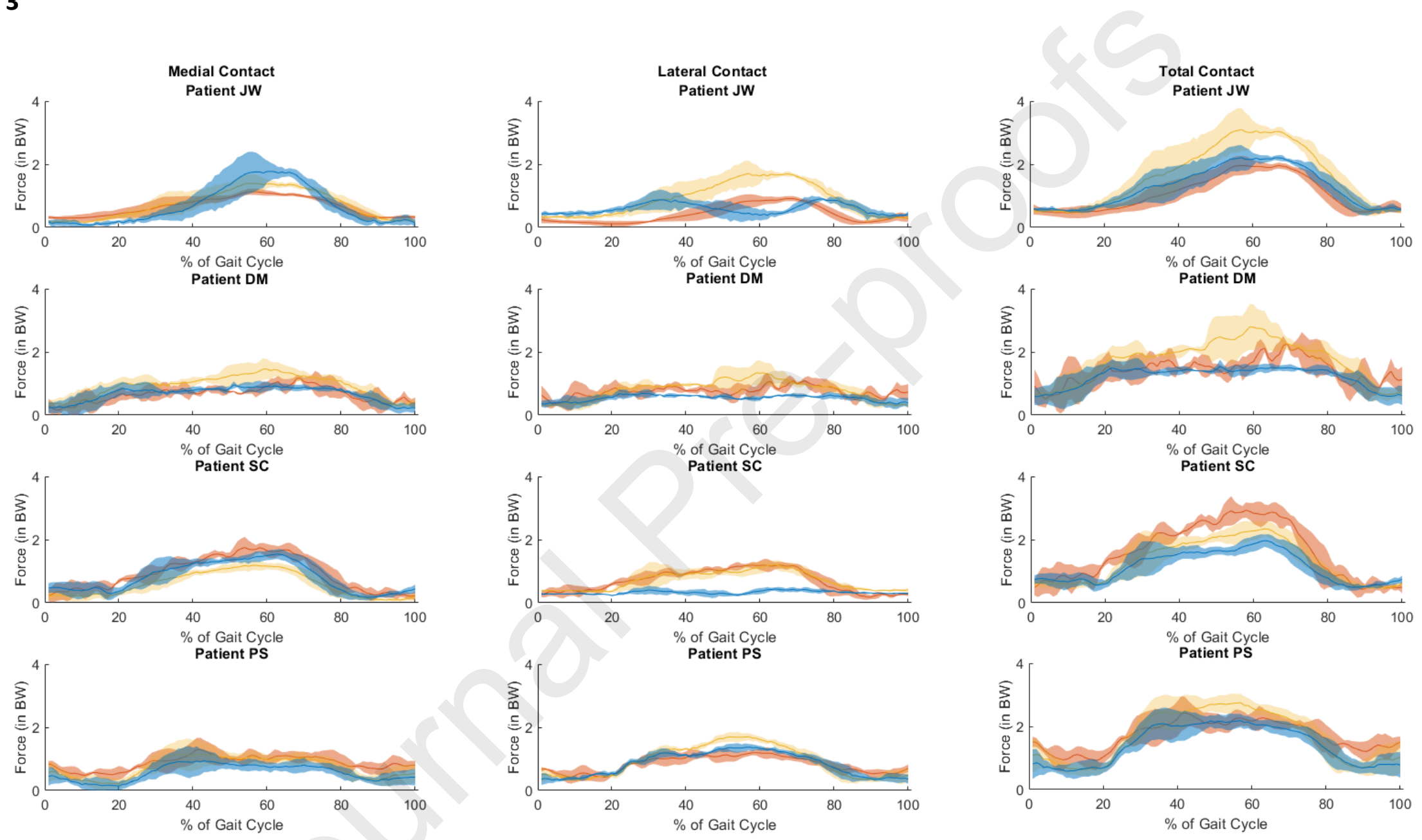
Table 1

\begin{tabular}{lcccc}
\hline & JW & DM & SC & PS \\
\hline Gender & Male & Male & Female & Male \\
Prosthesis side & Right & Right & Left & Left \\
$\begin{array}{l}\text { Age (years, at the time of fluoroscopy) } \\
\text { Months post-operative (months, at the time }\end{array}$ & 80 & 79 & 65 & 84 \\
of fluoroscopy) & 8 & 7 & 5 & 12 \\
Age (years, at the time of motion capture) & 83 & 83 & 68 & 86 \\
Mass $(\mathrm{kg})$ & 68 & 70 & 79 & 75 \\
Height $(\mathrm{m})$ & 1,66 & 1,7 & 1,63 & 1,8 \\
BMI $\left(\mathrm{kg} / \mathrm{m}^{2}\right)$ & 25 & 24 & 30 & 23 \\
\hline
\end{tabular}


Table 2

\begin{tabular}{|c|c|c|c|c|c|c|c|c|c|}
\hline \multirow[b]{2}{*}{ Gait } & & \multicolumn{2}{|c|}{ JW } & \multicolumn{2}{|c|}{ DM } & \multicolumn{2}{|c|}{ SC } & \multicolumn{2}{|c|}{ PS } \\
\hline & & Generic & Subject-specific & Generic & Subject-specific & Generic & Subject-specific & Generic & Subject-specific \\
\hline Medial & $1^{\text {rst peak }(B W)}$ & $0.35(0.12)$ & $0.07(0.13)$ & $0.87(0.30)$ & $0.62(0.29)$ & $-0.02(0.14)$ & $0.14(0.15)$ & $0.56(0.21)$ & $0.51(0.20)$ \\
\hline contact & $2^{\text {nd }}$ peak (BW) & $-0.22(0.15)$ & $-0.38(0.11)$ & $0.34(0.27)$ & $0.14(0.26)$ & $0.24(0.08)$ & $0.52(0.07)$ & $0.32(0.19)$ & $0.33(0.19)$ \\
\hline force & RMSE (BW) & $0.26(0.03)$ & $0.27(0.02)$ & $0.50(0.07)$ & $0.39(0.07)$ & $0.26(0.03)$ & $0.32(0.05)$ & $0.31(0.03)$ & $0.30(0.03)$ \\
\hline & $R^{2}$ & $0.86(0.03)$ & $0.87(0.03)$ & $0.77(0.10)$ & $0.76(0.10)$ & $0.75(0.05)$ & $0.76(0.06)$ & $0.87(0.03)$ & $0.88(0.02)$ \\
\hline Lateral & $1^{\text {rst peak }(B W)}$ & $-0.21(0.14)$ & $-0.31(0.13)$ & $-0.65(0.26)$ & $-0.74(0.23)$ & $-0.33(0.10)$ & $-0.58(0.09)$ & $-0.39(0.12)$ & $-0.40(0.13)$ \\
\hline contact & $2^{\text {nd }}$ peak (BW) & $-0.31(0.04)$ & $-0.30(0.04)$ & $-0.92(0.10)$ & $-0.87(0.12)$ & $0.15(0.10)$ & $-0.63(0.04)$ & $-0.35(0.11)$ & $-0.41(0.12)$ \\
\hline force & $R M S E(B W)$ & $0.32(0.02)$ & $0.30(0.03)$ & $0.56(0.12)$ & $0.55(0.13)$ & $0.28(0.03)$ & $0.44(0.04)$ & $0.45(0.01)$ & $0.50(0.01)$ \\
\hline & $R^{2}$ & $0.12(0.06)$ & $0.46(0.08)$ & $0.12(0.10)$ & $0.20(0.18)$ & $0.53(0.06)$ & $0.54(0.05)$ & $0.10(0.07)$ & $0.05(0.03)$ \\
\hline Total & $1^{\text {rst peak }(B W)}$ & $0.10(0.07)$ & $-0.19(0.07)$ & $0.12(0.58)$ & $-0.26(0.54)$ & $-0.69(0.28)$ & $-0.71(0.24)$ & $-0.32(0.19)$ & $-0.48(0.18)$ \\
\hline contact & $2^{\text {nd }}$ peak (BW) & $-0.55(0.18)$ & $-0.70(0.11)$ & $-0.62(0.34)$ & $-0.75(0.32)$ & $0.39(0.19)$ & $-0.14(0.11)$ & $0.02(0.17)$ & $-0.05(0.15)$ \\
\hline force & RMSE (BW) & $0.46(0.02)$ & $0.52(0.04)$ & $0.48(0.13)$ & $0.48(0.12)$ & $0.41(0.04)$ & $0.40(0.06)$ & $0.27(0.03)$ & $0.32(0.03)$ \\
\hline & $R^{2}$ & $0.75(0.03)$ & $0.82(0.04)$ & $0.71(0.14)$ & $0.74(0.13)$ & $0.78(0.04)$ & $0.82(0.03)$ & $0.92(0.01)$ & $0.92(0.01)$ \\
\hline $\begin{array}{c}\text { Musculo- } \\
\text { tendon } \\
\text { force }\end{array}$ & $C(\%)$ & $66.3(3.0)$ & $70.0(5.0)$ & $59.4(3.0)$ & $61.2(2.5)$ & $64.3(0.7)$ & $65.1(1.5)$ & $58.7(2.7)$ & $61.2(2.2)$ \\
\hline $\begin{array}{l}\text { Marker } \\
\text { residual }\end{array}$ & $R M S E(m m)$ & $14(1)$ & $16(1)$ & $24(1)$ & 21 (1) & $10(1)$ & $18(1)$ & $12(1)$ & $10(1)$ \\
\hline
\end{tabular}




\section{Table 3}

\begin{tabular}{|c|c|c|c|c|c|c|c|c|c|}
\hline \multirow[b]{2}{*}{ Squat } & & \multicolumn{2}{|c|}{ JW } & \multicolumn{2}{|c|}{ DM } & \multicolumn{2}{|c|}{ SC } & \multicolumn{2}{|c|}{ PS } \\
\hline & & Generic & Subject-specific & Generic & Subject-specific & Generic & Subject-specific & Generic & Subject-specific \\
\hline Medial & Peak (BW) & $0.35(0.06)$ & $0.84(0.17)$ & $0.32(0.28)$ & $-0.22(0.13)$ & $-0.70(0.10)$ & $-0.37(0.11)$ & $-0.01(0.05)$ & $-0.25(0.06)$ \\
\hline contact & $R M S E(B W)$ & $0.20(0.04)$ & $0.39(0.08)$ & $0.34(0.08)$ & $0.19(0.03)$ & $0.39(0.03)$ & $0.24(0.02)$ & $0.24(0.03)$ & $0.34(0.03)$ \\
\hline force & $R^{2}$ & $0.94(0.05)$ & $0.80(0.10)$ & $0.66(0.14)$ & $0.58(0.25)$ & $0.91(0.04)$ & $0.86(0.06)$ & $0.62(0.34)$ & $0.62(0.35)$ \\
\hline Lateral & Peak (BW) & $0.89(0.17)$ & $-0.01(0.09)$ & $0.20(0.33)$ & $-0.54(0.12)$ & $-0.05(0.06)$ & $-0.85(0.04)$ & $0.46(0.02)$ & $0.12(0.07)$ \\
\hline contact & RMSE (BW) & $0.57(0.07)$ & $0.42(0.05)$ & $0.28(0.02)$ & $0.28(0.04)$ & $0.17(0.02)$ & $0.51(0.03)$ & $0.30(0.06)$ & $0.20(0.05)$ \\
\hline forcet & $R^{2}$ & $0.83(0.07)$ & $0.03(0.03)$ & $0.35(0.22)$ & $0.28(0.21)$ & $0.79(0.04)$ & $0.16(0.20)$ & $0.78(0.10)$ & $0.77(0.10)$ \\
\hline Total & Peak (BW) & $1.27(0.18)$ & $0.29(0.03)$ & $0.59(0.61$ & $-0.76(0.13)$ & $-0.78(0.05)$ & $-1.22(0.10)$ & $0.31(0.20)$ & $-0.25(0.17)$ \\
\hline contact & $R M S E(B W)$ & $0.72(0.12)$ & $0.27(0.06)$ & $0.57(0.12)$ & $0.37(0.10)$ & $0.47(0.04)$ & $0.68(0.01)$ & $0.42(0.08)$ & $0.40(0.05)$ \\
\hline force & $R^{2}$ & $0.95(0.01)$ & $0.95(0.01)$ & $0.57(0.17)$ & $0.53(0.28)$ & $0.89(0.04)$ & $0.84(0.06)$ & $0.80(0.11)$ & $0.82(0.13)$ \\
\hline $\begin{array}{c}\text { Musculo- } \\
\text { tendon } \\
\text { force }\end{array}$ & $C(\%)$ & $71.6(1.0)$ & $73.2(9.2)$ & $66.7(4.8)$ & $61.9(4.7)$ & $73.2(6.3)$ & $65.5(3.1)$ & $63.5(3.1)$ & $65.5(4.1)$ \\
\hline $\begin{array}{l}\text { Marker } \\
\text { residuals }\end{array}$ & RMSE $(\mathrm{mm})$ & $22(1)$ & $14(2)$ & $37(1)$ & $24(1)$ & $21(1)$ & $18(1)$ & $21(1)$ & $27(1)$ \\
\hline
\end{tabular}




\section{Supplementary Table S1}

See xls file. 


\section{Supplementary Figure S1}
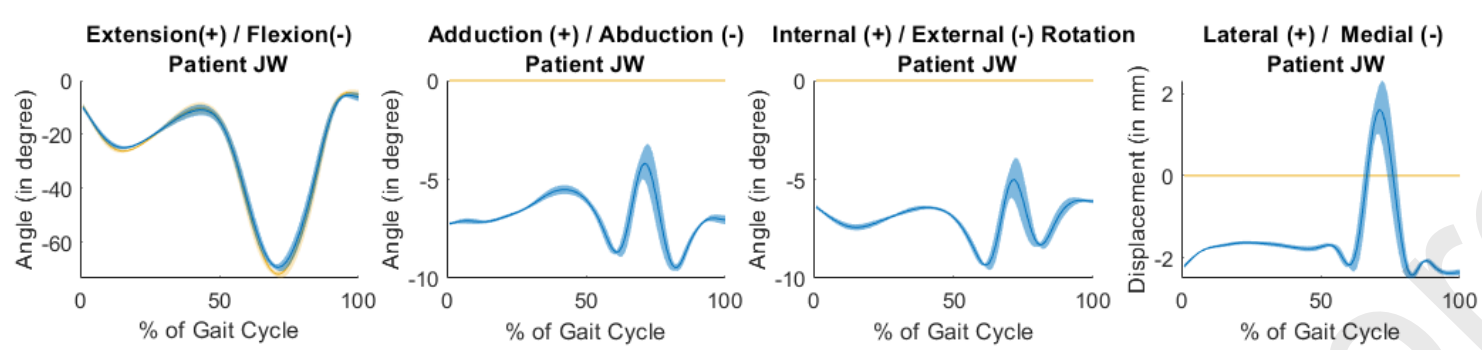

Anterior (+) / Posterior (-)
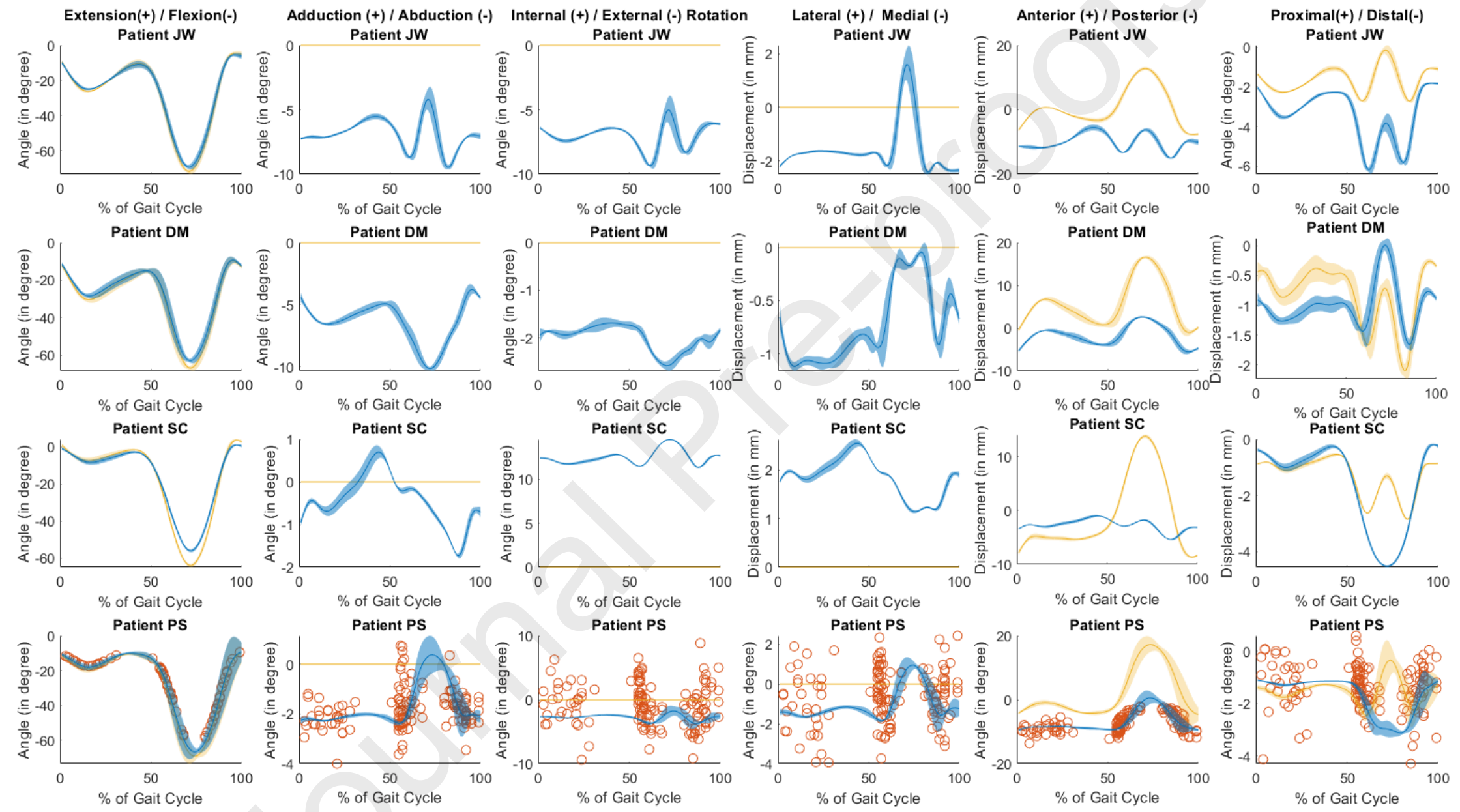


\section{Supplementary Figure S2}
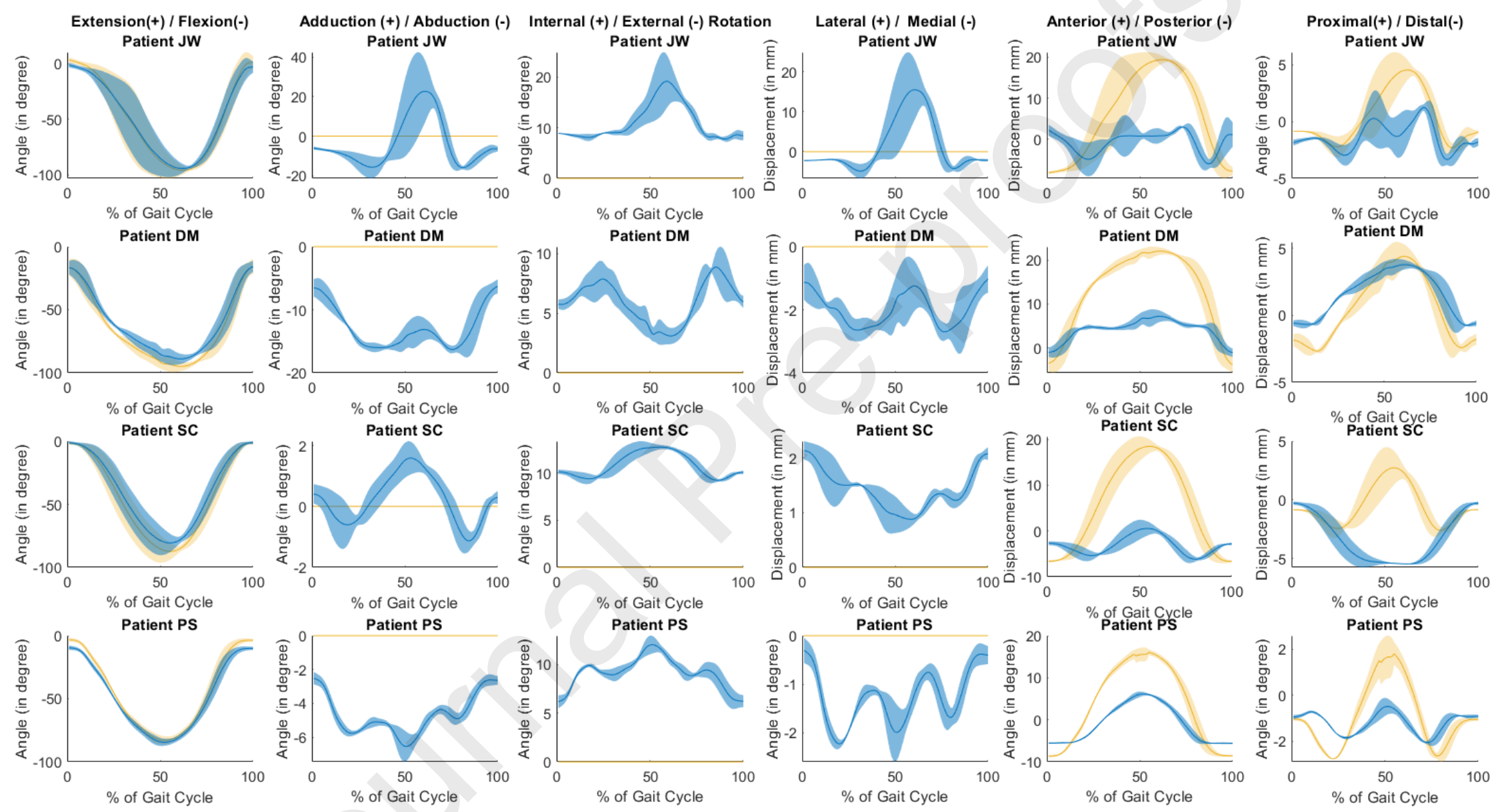